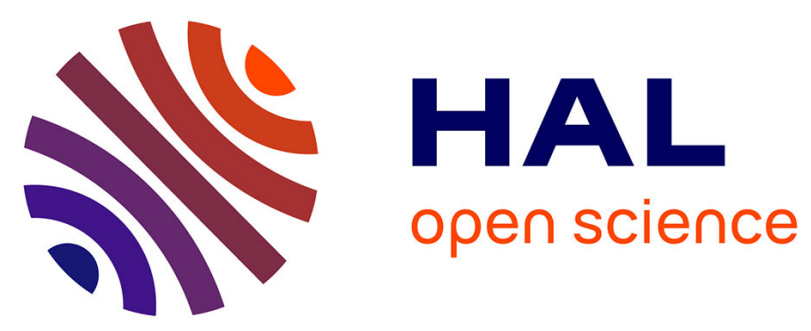

\title{
Séparer ou périr : conception et pratique du couloir réservé à Paris (1960-1975)
}

Arnaud Passalacqua

\section{To cite this version:}

Arnaud Passalacqua. Séparer ou périr : conception et pratique du couloir réservé à Paris (1960-1975).

Mélanges de l'École française de Rome - Italie et Méditerranée, 2008, 120 (1), pp.59-76. hal-01374127

\section{HAL Id: hal-01374127 \\ https://hal-univ-paris.archives-ouvertes.fr/hal-01374127}

Submitted on 29 Sep 2016

HAL is a multi-disciplinary open access archive for the deposit and dissemination of scientific research documents, whether they are published or not. The documents may come from teaching and research institutions in France or abroad, or from public or private research centers.
L'archive ouverte pluridisciplinaire $\mathbf{H A L}$, est destinée au dépôt et à la diffusion de documents scientifiques de niveau recherche, publiés ou non, émanant des établissements d'enseignement et de recherche français ou étrangers, des laboratoires publics ou privés. 


\title{
Séparer ou périr \\ Conception et pratique du couloir réservé à Paris (1960-1975)
}

\author{
Arnaud PassalacQua
}

Depuis la sortie de la Deuxième Guerre mondiale, et le développement de l'automobile individuelle qui l'a suivie, le réseau d'autobus parisien s'est engagé dans un long mouvement d'enlisement $^{1}$. Les statistiques annuelles de la RATP révèlent que sa vitesse commerciale à la pointe du soir a chuté entre 1952 et 1973 de 13,3 à $9,8 \mathrm{~km} / \mathrm{h}$ intra muros et de 18,1 à $13,6 \mathrm{~km} / \mathrm{h}$ extra muros $^{2}$. Les kilomètres perdus en raison de la congestion dépassent un million en $1967^{3}$. Pour maintenir le service, la RATP a dû acquérir près de 50 autobus par an de 1952 à $1968^{4}$. C'est donc dans un cercle vicieux qu'entre le système de transport en commun de surface, ce que dénoncent ouvertement plusieurs acteurs, extérieurs, comme Alfred Sauvy, ou au sein même du monde décisionnaire, comme le préfet de police Maurice Grimaud 5 . Les intérêts opposés sont clairement identifiés et mis en scène à la télévision, comme dans Cinq colonnes à la une en $1962^{6}$. La situation se trouve également dénoncée par les analystes de la forme urbaine et spécialistes des transports, comme Pierre Merlin, lorsqu'il décrit la vie fictive d'un couple de ban-

1. Nous employons ici plusieurs acronymes désignant les dépôts d'archives consultés: Archives de la Préfecture de police (APP), archives conservées à la médiathèque de la RATP (ARATP), Forum des images (FI) et National Archives de Kew Gardens (Royaume-uni) (NA).

2. Voir les Statistiques annuelles de la RATP. Les estimations de la baisse de vitesse varient selon les sources qui ne s'intéressent pas toutes à la même période, ne distinguent pas toutes entre Paris et la banlieue ou n'envisagent pas toutes la vitesse à l'heure de pointe du soir. Ainsi Louis Servant estime que la vitesse moyenne des autobus parisiens est passée entre 1952 et 1972 de 13,2 à 9,8 km/h (L. Servant, "L'amélioration des transports urbains", dans Notes et études documentaires, 4473, 1978, p. 11). Pour leur part, JeanClaude Ziv et Charles Napoléon détaillent la baisse de vitesse : en banlieue, de 1965 à 1968, elle est passée de 15,4 à lieusards: l'autobus y est «direct, confortable, mais plus lent et surtout irrégulier ${ }^{7}$ ».

Face à cette situation, qui touche aussi bien Paris que Londres et les villes de province et se traduit par des déficits financiers croissants, les pouvoirs publics ont expérimenté depuis le début des années 1960 différentes innovations, notamment l'idée d'exploiter des autobus de petit gabarit, les bus bleus, sans véritable succès. Ce n'est qu'en 1964, avec l'introduction d'un objet nouveau, le couloir réservé, que la logique alors à l'œuvre put être inversée et que le réseau d'autobus connut un certain renouveau. C'est une analyse de cet objet que nous proposons ici, dans sa conception, sa mise en place et son adoption par la population parisienne, autant que dans ce qu'il révèle des tensions en jeu sur l'espace public et politique du Paris des années 1960-1970.

Le couloir d'autobus s'avère effectivement être un objet cristallisant particulièrement les différentes forces en présence. À travers un aménagement souvent sommaire, un simple trait de peinture, c'est l'ensemble du système de la

$14,4 \mathrm{~km} / \mathrm{h}$; à Paris, de 10,4 à $9,8 \mathrm{~km} / \mathrm{h}$ (C. Napoléon et J.-C. Ziv, Le transport urbain, un enjeu pour les villes, Paris, 1981, p. 40).

3. "Quelques observations sur la circulation des autobus dans Paris", mars 1968 (ARATP, IR 184).

4. Idem.

5. Voir A. Sauvy, Les quatre roues de la fortune, Essai sur l'automobile, Paris, 1968, p. 157. Comme l'explique Maurice Grimaud en 1968, "cette clientèle perdue va en grande partie à la voiture particulière accroissant d'autant l'encombrement des rues donc le ralentissement de la circulation générale et donc le retard des autobus. C'est un cercle infernal!» (M. Grimaud, "La circulation à Paris», dans Liaisons, 150, 1968, p. 4).

6. Voir FI, Les troubles de la circulation.

7. P. Merlin, Vivre à Paris 1980, Paris, 1971, p. 148. 
chaussée parisienne, et plus précisément de l'autobus, dont la conception évolue. C'est alors la priorité accordée aux transports en commun, longtemps réclamée par certains élus et par la RATP, qui se trouve symboliquement inscrite sur l'espace public, sans toutefois faire oublier que cette même société parisienne achève un périphérique, construit de multiples autoroutes et s'équipe d'un métro régional (RER). Dès lors l'efficacité essentielle des couloirs n'est peut-être pas l'amélioration de vitesse et de régulation qu'ils ont permise, mais l'avancée qu'ils ont marquée en faveur de l'autobus dans un paysage politique, technique et culturel, où le recours aux modes dominants, automobile individuelle et réseau ferré, faisait jusque-là figure de solution unique.

\section{GÉNÉALOGIE DE «LA THÉORIE DU DOMAINE RÉSERVÉ8}

Il existe une double généalogie du couloir d'autobus, celle, concrète, d'un espace réservé à l'un des modes, et celle, plus théorique, d'une priorité en faveur des transports en commun?. Tandis que dès le début du XIX ${ }^{\mathrm{e}}$ siècle, les trottoirs ont permis d'accueillir, des piétons, c'est l'installation des rails de tramway qui marque le premier pas vers leur éviction de l'espace de la rue ${ }^{10}$. Cette régulation résulte simultanément du rejet des piétons par les tramways et de leur choix en faveur d'un espace plus propre et sûr que la chaussée. Mais c'est au tout début du $\mathrm{XX}^{\mathrm{e}}$ siècle que prend forme la théorie de séparation des flux qui domine peu à peu la conception de la circulation urbaine, au moins jusqu'aux années 1970, notamment sous l'influence d'Eugène Hénard, à Paris ${ }^{11}$. Le journal satirique L'assiette au beurre ne pressentait-il pas, dès 1908, une chaussée réservée aux tout nouveaux autobus, reléguant les piétons sur une dalle latérale (fig. 1) ${ }^{12}$ ?

8. La théorie du domaine réservé, dans Combat, 16 janvier 1964.

9. Il existe peu de travaux sur l'histoire des couloirs réservés aux autobus, ceux de Mathieu Flonneau faisant office de premiers fondements (M. Flonneau, L'automobile à la conquête de Paris, 1910-1977. Formes urbaines, champs politiques et représentations, thèse de doctorat d'histoire, sous la direction d'Antoine Prost, Université Paris I Panthéon-Sorbonne, 2002, II, p. 514-519).

10. Voir M. Garden, Histoire de la rue, dans Pouvoirs, 116, 2006 p. 13. Les premiers trottoirs parisiens sont ceux du quartier de l'Odéon, réalisés dans les années 1780. Maurice Garden

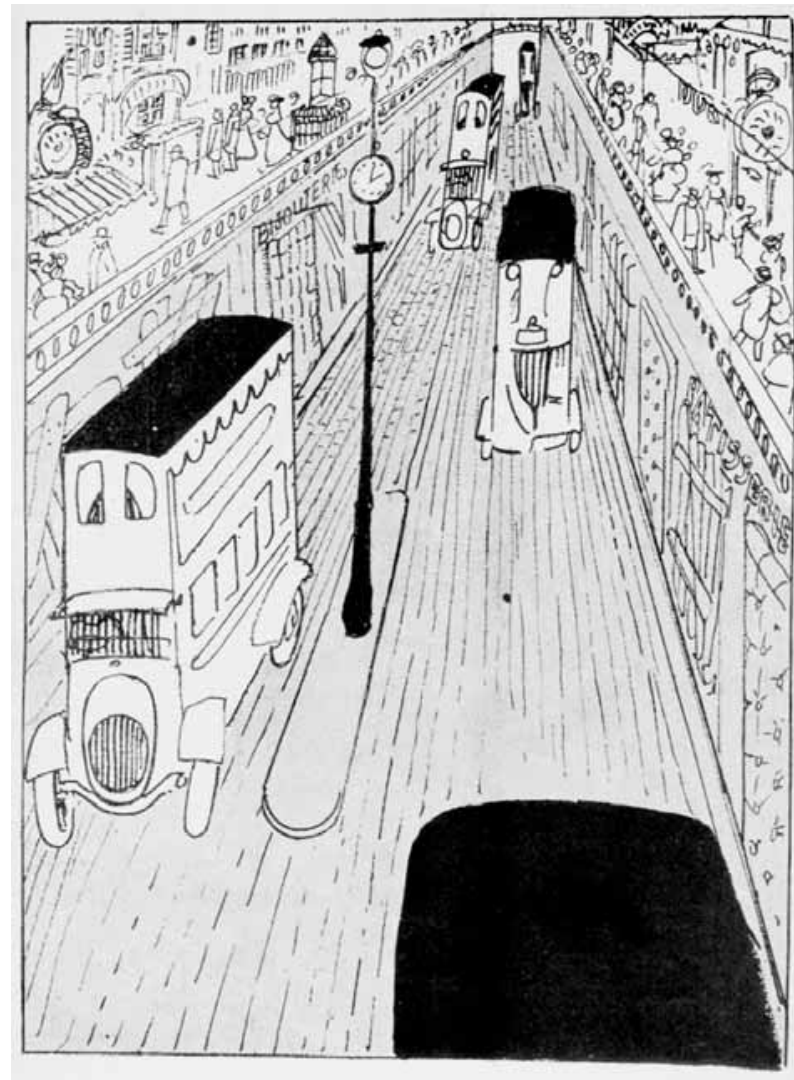

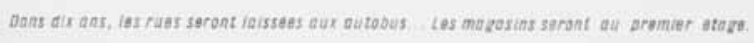

Fig. 1 - «Dans dix ans, les rues seront laissées aux autobus... Les magasins seront au premier étage». L'assiette au beurre, Édition du 7 mars 1908.

Dès les années 1920, les premières mesures issues de ce courant sont appliquées aux ChampsÉlysées, selon une logique de rejet des véhicules plus lents ou de transport en commun sur le côté droit de la chaussée. L'autobus a ainsi rejoint «les cycles, tandems, triporteurs et les catégories de voitures attelées", autant de véhicules condamnés par la modernité automobile à ne plus occuper que le bas-côté13. Mais cette distinction était bien plus destinée à dégager la chaussée centrale pour

explique par ailleurs que la séparation des fonctions s'est engagée avec la création de halles, marchés et abattoirs, afin de désengorger un espace public encombré par les activités marchandes (ibid., p. 10-11).

11. Sur ce point, voir C. Studény, L'invention de la vitesse, France, $X V I I I^{e}-X X^{e}$ siècle, Paris, 1995, p. 112-113.

12. L'assiette au beurre, 7 mars 1908. Le processus de motorisation des omnibus avait été engagé depuis 1905-1906.

13. Ordonnance du 31 octobre 1924. Voir M. Desportes, Paysages en mouvement, Paris, 2005, p. 217-218. 
la circulation des automobiles individuelles qu'à laisser aux autobus un espace où ils pussent plus aisément évoluer. Leur relégation était d'ailleurs nettement plus liée aux arrêts qu'ils devaient marquer qu'à des considérations sur leur vitesse. Dès lors, l'artère parisienne la plus prestigieuse a alimenté l'image d'une chaussée séparée, dans les pratiques et non matériellement, pour des raisons très différentes de celles à l'origine des couloirs des années 1960. Dans ce cas, la forme préexiste donc à la conception même de l'objet ${ }^{14}$.

Bien plus tard, au cours des mois précédant immédiatement l'ouverture du premier couloir en 1964, la Préfecture de police commençait à régulariser la situation de nombreux terminus où la RATP s'était octroyé différents espaces pour le stationnement et les manœuvres de ses véhicules, en apposant des panneaux illégaux. Alors que depuis la guerre, les services de police s'étaient montrés intransigeants sur ces pratiques courantes de la RATP, en 1963, ils entérinent cette appropriation de l'espace par l'exploitant en lui en réservant officiellement l'usage en 14 points de la capitale ${ }^{15}$. Ce mouvement prépare donc la décision dans sa forme concrète.

Par ailleurs, l'idée d'une priorité donnée aux transports en commun n'est pas neuve. Comme l'a montré Mathieu Flonneau, le conseiller Georges Contenot, élu républicain-national du quartier de la porte Saint-Martin, semble être le premier à avoir milité en faveur d'une "priorité

14. L'idée de séparation des flux peut également se trouver dans l'attribution du sous-sol parisien au transport collectif et du sol au transport individuel, idée avancée lors de la suppression des tramways dans l'entre-deux-guerres et qui ne simplifia pas le positionnement des autobus depuis lors. Cette idée a par exemple été débattue lors de l'élection municipale parisienne de 2008. La candidate conservatrice Françoise de Panafieu promouvait ainsi le transport ferré souterrain d'une part et le transport individuel en surface, d'autre part.

15. Arrêté préfectoral du 9 avril 1963 et lettre du directeur de la circulation, des transports et du commerce au directeur général de la RATP du 31 mai 1963 (voir les cartons ARATP, IR 49 et 1R 184).

16. Voir M. Flonneau, L'automobile à la conquête de Paris, 1910$1977 \ldots$ cit., I, p. 248-249.

17. «La question de principe de l'existence des autobus ne saurait être discutée, au contraire", affirmait-il (ibid., I, p. 377).

18. Ibid., I, p. 375-376. Pierre Ruais était président du Conseil municipal de Paris et de l'Office régional des transports parisiens (ORTP). Il est très clair dans la révolution copernicienne à opérer : «c'est ainsi qu'apparaît la double notion d'itinéraire privilégié, d'une part et de réseau principal, aux voitures de transport en commun", dès 192916. L'autobus disposait donc de longue date sinon d'une priorité sur l'espace politique, du moins d'un affichage net de nombreux élus en sa faveur. Après la guerre, Bernard Lafay, président centriste du Conseil municipal de Paris, avait affirmé son attachement à l'existence du réseau d'autobus $^{17}$. En 1955, Pierre Ruais, rapporteur gaulliste des Perspectives nouvelles sur les transports en commun de voyageurs dans la région parisienne, prônait une politique volontariste: "faut-il abandonner les autobus à leur sort et se résigner à l'embouteillage spécialement dans Paris? Bien au contraire, il faut prévenir cette situation et revoir la manière d'utiliser les autobus en fonction du développement exceptionnel des moyens de transport privés ${ }^{18} "$. L'idée de discriminer la voirie en faveur des autobus naît alors logiquement sous

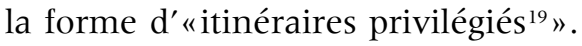

En octobre 1959, le Conseil municipal vote à l'unanimité une délibération dont l'article 4 précise que "les problèmes de la circulation doivent être réglés de manière à accorder toutes facilités aux moyens de transports collectifs et à décourager la multiplication des moyens de transports particuliers ${ }^{20} »$. Les propositions alors avancées ne sont toutefois pas retenues, malgré les études menées par la RATP en vue de comprendre les difficultés de son réseau et d'en améliorer les performances. En réalité, les pratiques d'aménagement ne reflètent pas les discours en faveur d'une prio- d'autre part, ce qui n'exclut nullement que sur les itinéraires qui pourront être privilégiés, sans présenter la largeur ou le dégagement optimum, la RATP doive faire l'effort d'un matériel moins encombrant et adapté tout aussi bien à tous les itinéraires à voies étroites ou privilégiées. On sera enfin amené à établir les priorités et utilités respectives des investissements au titre des transports publics et des investissements au titre de la circulation et à accorder souvent la priorité aux premiers au détriment des seconds» (ibid., p. 376).

19. Notons par ailleurs que Maurice Grimaud, alors préfet de police, explique ainsi la naissance des couloirs d'autobus : "[Le réseau d'autobus offre] une desserte assez fine bien adaptée aux besoins des usagers. Le drame est que ces autobus s'engluent chaque année davantage dans la circulation générale. On a bien essayé les minibus ou autobusbleus; ils n'ont pas progressé beaucoup plus vite et le problème reste entier. La solution, Christophe Colomb l'eût trouvée : puisque l'autobus n'avance plus dans la circulation générale, eh bien il faut l'en retirer. Ainsi sont nés les "couloirs réservés"» (M. Grimaud, La circulation à Paris... cit., p. 7).

20. Liaisons, 3 février 1964. 
rité donnée aux transports publics. Dans les documents de planification émis par le District, l'autobus fait figure de troisième et dernier «réseau secondaire de liaisons", après le métro et les parkings de petite taille; lorsque ses ingénieurs traitent de séparation des flux, c'est plus de la distinction entre trafic de transit et trafic local, même si, comme ils l'écrivent en 1963, «il n'est pas exclu, enfin, que des voies existantes puissent être spécialisées soit pour le trafic des autobus, soit pour celui des piétons ${ }^{21}$ ".

Si la Préfecture de police cède enfin aux demandes de la RATP, c'est probablement pour des raisons financières, l'exploitant, contraint d'accroître son parc au fur et à mesure que diminue la vitesse commerciale, se trouvant dans une situation difficile, qui entretenait d'ailleurs une crise de confiance entre lui et l'administration ${ }^{22}$. L'idée que tout ne pouvait pas être fait en faveur du RER, dont les travaux rencontraient d'ailleurs des difficultés, sans améliorer également l'offre des modes existants est probablement présente dans la tête de certains acteurs, sans que cela soit très explicite. Il est plus net que les idées keynésiennes dominantes ont incité à considérer tout investissement en faveur des transports comme pouvant alimenter l'activité économique de la capitale.

$C^{\prime}$ est donc le contexte et non l'argumentation qui évolue. Les éléments mis en avant en faveur des transports publics sont sensiblement identiques à ceux déployés au cours de l'entre-deuxguerres, notamment la comparaison, devenue progressivement classique, de la surface occupée par chacun, à bord de son automobile ou à bord

21. Avant-projet de programme duodécennal pour la région de Paris, Paris, 1963, p. 103.

22. Voir A. Delassus, La deuxième génération des autobus à impériale et des autobus à plate-forme arrière (1968-2002), Approche historique d'un phénomène urbain, social et culturel parisien de la fin $d u X X^{e}$ siècle, maîtrise d'histoire, sous la direction d'Annie Fourcaut et de Mathieu Flonneau, Université Paris I Panthéon-Sorbonne, 2005, p. 41.

23. L'expérience des "pistes" pour autobus est concluante déclare la RATP après quelques jours d'essai sur les quais, dans Combat, 20 janvier 1964. L'argumentaire fondé sur la surface occupée par chacun sur l'espace public est une constante des documents produits sur le sujet jusqu'à la fin du $\mathrm{XX}^{\mathrm{e}}$ siècle (voir par exemple, FI, Paris, ville à deux vitesses). Le chiffre avancé par le Plan de déplacements urbains en 2000 selon lequel $94 \%$ de la voirie serait réservé à l'automobile est directement issu de cette logique (Plan de déplacements urbains de la Région Île-de-France, Paris, 2000, p. 21).

24. La lutte contre les privilèges des couloirs de circulation. Le SNA attaque le Préfet de Police en excès de pouvoir, dans L'auto- des autobus : 1,7 personne par véhicule particulier contre 50 à 70 personnes à bord des autobus en période de pointe en $1964^{23}$. Un argument en partie biaisé puisque la capacité de l'autobus est prise à son maximum, alors que celle de l'automobile est constatée sur la voirie parisienne, ce que ne manquent pas de dénoncer les représentants des intérêts automobiles, comme L'auto-journal, mettant en avant l'occupation réelle des autobus, autour de 20 personnes par véhicule ${ }^{24}$. Enfin, en ce début des années 1960, les voies réservées aux autobus existent outre-Atlantique et en Europe ${ }^{25}$. Comme l'explique la RATP, en suivant cette voie, Paris ne ferait donc que combler son retard.

Avec le travail en cours de la commission ministérielle Lapébie, chargée de proposer des solutions en vue d'une renaissance du réseau d'autobus, le moment était donc favorable à l'engagement d'une politique tranchant entre des intérêts d'abord communs, puis qui se sont opposés, bien que les pouvoirs publics aient tenté de les concilier : ceux de l'automobile individuelle et ceux de l'autobus ${ }^{26}$. Le couloir en est l'expression la plus visible, rendant la lecture des intérêts particuliers directement possible sur l'espace public.

\section{LES PREMIERS COULOIRS}

La première bande de roulement réservée aux autobus est donc ouverte le 15 janvier 1964 par décision de Maurice Papon, préfet de police, sur les quais du Louvre et de la Mégisserie, entre les ponts du Carrousel et au Change ${ }^{27}$. Délimité par

journal, 5 mars 1964.

25. Les travaux de la commission ministérielle Lapébie ont fait le point sur l'état des bandes réservées aux autobus dans les principales agglomérations mondiales au milieu des années 1960. La ville la mieux équipée est alors Stockholm, avec 8 $\mathrm{km}$ de couloirs qui sont en fait des voies de tramway ouvertes à la circulation des autobus, suivie, en Europe, par Milan, avec près de $4 \mathrm{~km}$ de couloirs réservés en permanence. Seules huit villes européennes possèdent toutefois ce type d'équipement. Les États-Unis semblent mieux dotés. En revanche d'autres dispositifs sont développés dans de nombreuses villes, comme des signaux lumineux spéciaux, voire des voies exclusivement réservées aux autobus, à Aix-laChapelle, Bâle, Hanovre, Milan, Munich et Tel Aviv (voir le carton ARATP, IR 284).

26. Sur les travaux de la commission Lapébie en lien avec les couloirs réservés, voir le carton ARATP, 1R 284.

27. Quant au terme même de couloir, il a longtemps et uniquement désigné le couloir délimité par des barrières ou des chaînes canalisant les voyageurs en de multiples points 
une ligne continue jaune doublée d'une ligne discontinue permettant d'éventuels dépassements et d'une largeur de $3 \mathrm{~m}$, il est exclusivement dédié à la circulation des autobus de $8 \mathrm{~h}$ à $21 \mathrm{~h}$, ces derniers étant tenus d'y rouler, sauf en cas $\mathrm{d}^{\prime}$ encombrement ${ }^{28}$. Les 13 lignes d'autobus qui l'empruntent représentent 80 passages à l'heure en période de pointe, à rapporter à la circulation automobile quotidienne sur cet axe, de 40.000 véhicules ${ }^{29}$. Le choix du quai est directement lié à l'absence de commerces et riverains, permettant de simplifier l'exploitation de ce couloir, en évitant les contestations et les problèmes de livraison ${ }^{30}$.

Dès le premier jour surgissent les difficultés qui devinrent récurrentes sur ces questions : tourne-àdroite des voitures, traversées piétonnes non-protégées, positionnement des deux roues, tourne-àgauche des autobus... Le respect du nouvel équipement semble plutôt bon, même si des ambulances et véhicules d'urgence l'empruntent également ${ }^{31}$. Pour la RATP, "les résultats ont été spectaculaires": baisse de $75 \%$ des kilomètres perdus sur les six lignes parcourant le couloir dans toute sa longueur, augmentation de $157 \%$ de la vitesse des autobus à l'heure de pointe du $\operatorname{soir}^{32} \ldots$

Les chauffeurs de taxis s'étaient exprimés contre le projet, notamment du fait de l'encombrement accru sur la voirie laissée à la circula- tion courante mais en avaient aussitôt réclamé l'accès : "nous sommes nous aussi en quelque sorte, un service de transport en commun et un service public. Un seul taxi dans Paris vaut plusieurs voitures particulières ${ }^{33}$ ». Leur argument, mais également la volonté de remplir la bande de circulation, jugée trop vide lorsque la voirie courante est embouteillée, incitent le préfet de police, à ouvrir le couloir, à partir du 2 mars 1964, à "la circulation des véhicules de police, de gendarmerie, de secours contre l'incendie, des ambulances et des taxis ${ }^{34} »$. Même si, sur la bande de roulement, les taxis n'ont pas le droit de prendre en charge ou laisser descendre des voyageurs, ni de doubler d'autres véhicules, pour la RATP «les résultats marquent [alors] une nette régression ${ }^{35}$ ". Les 400 taxis à l'heure qui utilisent le couloir pendant l'après-midi l'engorgent et aggravent les problèmes de tourne-à-droite. En outre, les chauffeurs multiplient les dépassements illicites et changements de voies intempestifs, qui contribuent à brouiller la fluidité de l'axe. Cette ouverture aux taxis, si elle leur procure un avantage indéniable, s'opère nettement aux dépens des autobus $^{36}$. Leur vitesse baisse alors de $34 \%$ à l'heure de pointe du soir sur le tronçon concerné ${ }^{37}$. Néanmoins, assez paradoxalement, l'autobus se trouve simultanément un allié de cir- d'arrêt. La presse l'emploie dès le milieu des années 1960 pour désigner les nouveaux espaces réservés, si bien qu'il remplace rapidement les termes de bande de roulement ou de piste de roulement, initialement en usage à la RATP.

28. Ce nouvel objet impose quelques réaménagements d'arrêts. Classiquement, il s'agit d'abord d'un dispositif mis à l'essai. L'ouverture du couloir s'accompagne d'une présence policière renforcée pour veiller à sa bonne utilisation.

29. Les lignes concernées sont les lignes $21,24,27,58,67,69$, $72,73,74,75,76,81$ et 85 .

30. Voir la lettre du préfet de police au ministre des travaux publics et des transports du 31 décembre 1963 (ARATP, IR 184). La direction de la RATP aurait préféré une bande à contresens afin de retrouver des itinéraires dissociés par les sens uniques, notamment sur les Grands Boulevards, mais cette solution semblait trop ambitieuse aux services de la Préfecture de police (note RATP/R/14329 du 9 octobre 1963 [ARATP, 1R 284]).

31. Rapport de la RATP au sujet de la bande de roulement réservée aux autobus sur les quais de Seine rive droite, 11 février 1964 (ARATP, 1R 282).

32. Quelques observations sur la circulation des autobus dans Paris, mars 1968 (ARATP, IR 184).

33. L'expérience des "pistes" pour autobus est concluante déclare la RATP après quelques jours d'essai sur les quais... cit. Sur les taxis parisiens à l'époque, voir P. Laneyrie et J. Roux, Transport traditionnel et innovation technique. L'exemple du taxi en
France, dans Culture technique, 19, 1989, passim. Les chauffeurs n'hésitaient pas, en 1962, à se présenter comme étant "les vieux sages" (FI, Les troubles de la circulation).

34. Note RATP/RM/3003 du 26 février 1964 (ARATP, IR 184). Comme l'écrit le journal interne de la Préfecture de police, «aux heures de pointe, [la bande influe] sur le trafic général et, psychologiquement, elle [est] de mauvais effet sur les automobilistes ralentis dans leur progression" (Liaisons, 2 mars 1964).

35. Quelques observations sur la circulation des autobus dans Paris, mars 1968 (ARATP, 1R 184). Les modalités d'utilisation par les taxis furent conservées sur l'ensemble des couloirs réservés mais laissaient en suspens la question du cadre légal des montées et descentes des passagers de taxis.

36. En 1969, lors d'une visite de responsables londoniens, les experts de la RATP et de la Préfecture de police ne cachaient pas leur manque d'enthousiasme à propos de l'ouverture des couloirs aux taxis (Public transport and parking developments in Paris with particular reference to bus lanes - note of visit by G.L.C./L.T.B. offices in april 1969, avril 1969 [NA, MT/106/ 314]).

37. Le 4 décembre 1964, un feu spécifique au couloir a été installé pour faciliter le passage sur le pont Neuf des voitures, ce qui a encore ralenti les autobus. Par ailleurs, un léger report d'automobilistes sur le boulevard Saint-Germain est constaté. 
constance, le taxi, qui milite dès lors en faveur de la généralisation des couloirs.

Suite aux bons résultats du premier couloir réservé, la Préfecture de police et le Syndicat des transports parisiens (STP) en créent successivement d'autres à partir d'une liste dressée par la RATP, à Paris, puis en banlieue (fig. 2). Aguerrie, la puissance publique se lance dès 1965 dans l'ouverture d'un nouveau type d'équipement : le couloir à contresens, le premier se situant avenue Bosquet. Ce nouvel objet est toutefois beaucoup plus sensible aux problèmes de respect, puisque s'il est bloqué, l'autobus ne peut dépasser l'obstacle et l'itinéraire se trouve dévié. D'une façon générale, les gains de temps liés à la présence d'un couloir sont estimés à 30 à $70 \%$ selon les axes ${ }^{38}$. Le rythme de création reste toutefois faible jus- qu'aux années 1967-1968, quand Maurice Grimaud définit un "plan de priorité aux autobus» et décide de l'ouverture de couloirs ambitieux, rue de Rivoli, et, à contresens, sur les boulevards Saint-Michel et Saint-Germain (fig. 3).

En 1971-1972, ce même préfet de police relance cette politique en engageant un plan d'équipement en nouveaux couloirs à un rythme soutenu suivant une logique cohérente, dépassant celle du coup par coup jusque-là dominante et l'accompagnant d'une répression des pratiques illicites. Ce nouveau souffle est dû à la situation financière délicate de la RATP, fragilisée par le refus des collectivités publiques d'augmenter leur participation à son budget en 1971 et le report du relèvement tarifaire prévu au $1^{\text {er }}$ janvier $1971 \mathrm{du}$ fait de mouvements d'usagers. L'attention s'est

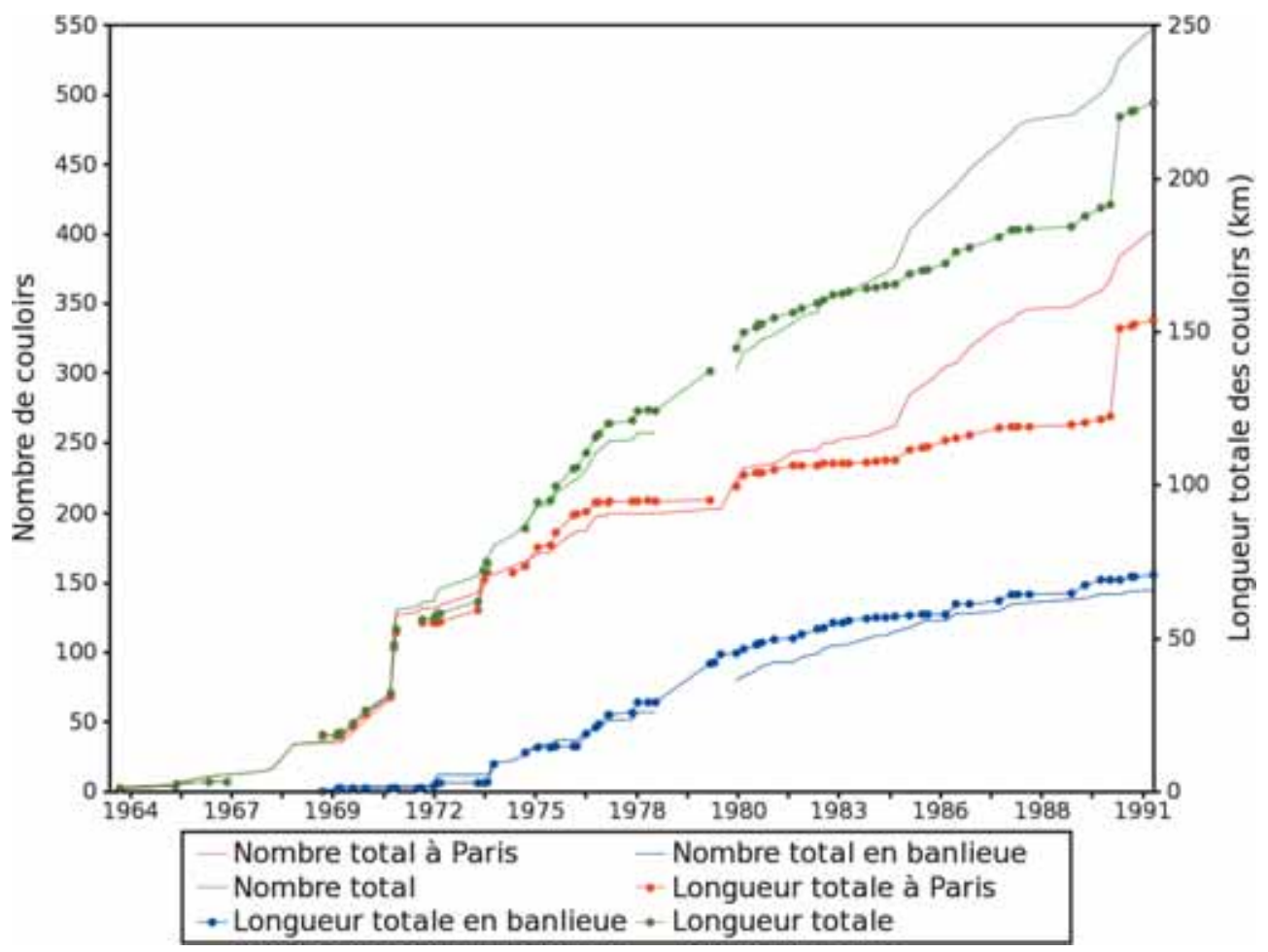

Fig. 2 - Évolution du nombre et de la longueur des couloirs réservés aux autobus (1964-1991). Statistiques annuelles de la RATP, Bulletin d'information et de documentation et Entre les lignes.

38. De nouveaux couloirs de circulation, dans Liaisons, 180, 1971, p. 16-22. À la fin de 1970, avec $25 \mathrm{~km}$ de couloirs et selon une étude de la RATP, le gain de vitesse de rotation serait de $5,2 \%$; entre 1968 et 1969, le nombre de kilomètres perdus pour difficultés de circulation a diminué de $8 \%$ sur les lignes les plus pourvues en couloirs alors qu'il a augmenté de $24 \%$ sur le réseau; simultanément, le nombre de retards supérieurs à 15 minutes a diminué de $4 \%$ sur ces mêmes lignes alors qu'il a augmenté de $9 \%$ sur le réseau (lettre du préfet de police au préfet de région du 5 février 1971 [APP, DA 830]). 

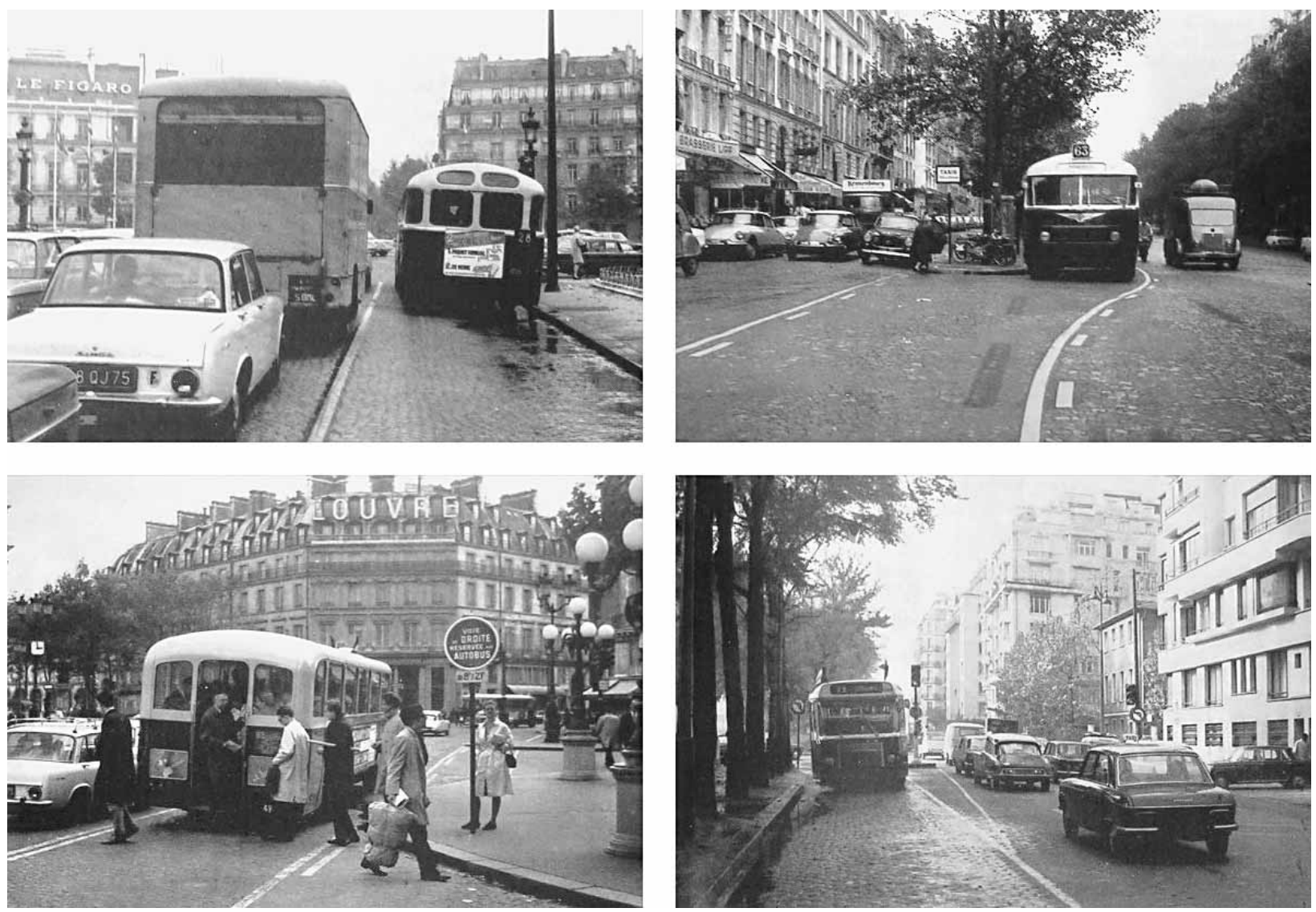

Fig. 3 - Quelques photos de couloirs à Paris en 1968. De haut en bas et de gauche à droite : avenue Franklin Roosevelt, boulevard Saint-Germain, avenue de l'Opéra et avenue du président Kennedy (couloir à contresens). Quelques observations sur la circulation des autobus dans Paris, mars 1968 (ARATP, 1R 184).

donc portée sur "l'environnement» dont un sousgroupe de travail est en charge au sein de la Préfecture de police ${ }^{39}$. Pour ce sous-groupe, il est très net que les intérêts des autobus s'opposent à ceux de l'automobile individuelle et que des mesures doivent être prises : stationnement payant et relèvement des amendes pour les voitures, couloirs réservés et tarifs abaissés pour les autobus. Comme l'écrit un de ses collaborateurs à Maurice Grimaud :

[Il y a] une vérité première à savoir que les autobus verront leur vitesse s'accroître dans la mesure même

39. D'après ce sous-groupe, pour maintenir la vitesse des autobus urbain $(10,81 \mathrm{~km} / \mathrm{h}$ en 1970), il faut créer $17 \mathrm{~km}$ de couloirs en 1971, $9 \mathrm{~km}$ en 1972, $9 \mathrm{~km}$ en 1973 et $9 \mathrm{~km}$ en 1974. En banlieue, pour maintenir une vitesse de $13,9 \mathrm{~km} / \mathrm{h}$ il faut en créer $10 \mathrm{~km}$ par an. Pour leur surveillance, il faudrait 1200 policiers supplémentaires pour en disposer un tous les $300 \mathrm{~m}$ de $8 \mathrm{~h}$ à $20 \mathrm{~h}$. Il faudrait ainsi enlever 1200 où la circulation des voitures privées diminuera. [...] L'objectif consiste donc à inciter les parisiens et surtout les habitants de la banlieue à se servir de moins en moins de leur voiture particulière. [il ne] semble pas absurde dans cette situation de "dos au mur» de proposer toutes solutions qui seraient de nature à ramener à "l'autobus" nombre d'anciens usagers qui depuis plusieurs années préfèrent se servir de leur voiture personnelle ${ }^{40}$.

Les couloirs se généralisent donc dans le paysage parisien, dépassant les $100 \mathrm{~km}$ en 1980, mais leurs horaires d'ouverture sont variables, ce qui voitures par jour, soit 10 fois plus que ce qui est pratiqué sur les zones concernées, ce qui nécessiterait 960 agents supplémentaires, des infrastructures et du matériel nouveaux (note «Budget de la RATP, accroissement de la vitesse des autobus» de Maxime Mignon au préfet de police, ca. 1971 [APP, DA 830]).

40. Ibidem. 
contribue à brouiller leur compréhension ${ }^{41}$. Chaque implantation nouvelle demande un travail très fin, prenant en compte les pratiques des différents acteurs présents sur l'espace public et impose ainsi aux concepteurs de développer une connaissance spécifique du territoire traversé, notamment des occupations temporaires de l'espace public, que ce soit par les livraisons ou les marchés.

Les responsables des transports londoniens sont très impressionnés par la visite qu'ils font en avril 1969 du système en place à Paris ${ }^{42}$. À leur retour, ils organisent d'ailleurs une conférence interne afin de présenter le cas parisien ${ }^{43}$. La différence principale avec le cas londonien est la largeur des voies parisiennes. Les couloirs réservés ayant été le plus souvent placés sur des axes à sens unique, il reste encore plusieurs files pour la circulation automobile. La Préfecture de police estime qu'il convient de laisser au moins trois voies, malgré des cas où il n'en reste que deux comme boulevard Saint-Michel ou avenue de l'Opéra. À leurs hôtes londoniens, les services de la Préfecture de police confient d'ailleurs leur surprise du succès des couloirs, quand ceux de la RATP parlent de leurs difficultés à les étendre en banlieue. En retour, Paris copia les dispositifs de signalisation des couloirs londoniens lors de l'établissement des «Lignes pilotes» en $1973^{44}$.

Les premiers couloirs londoniens, dans le sens et à contresens de la circulation, sont ouverts en 1968-1969, avec, le plus souvent un fonctionnement uniquement aux heures de pointe du matin en semaine ${ }^{45}$. Conséquence de leur succès, Londres se lance dans un programme d'équipement de ses principales artères en couloirs d'au-

41. Certains sont réservés en permanence, d'autres uniquement l'après-midi afin de permettre les livraisons le matin, d'autres, enfin, aux heures de pointe du matin et du soir. La direction de la RATP souhaite alors la généralisation de l'ouverture des couloirs à la journée entière. Pour un plan des couloirs en 1976, voir M. Flonneau, L'automobile à la conquête de Paris, 1910-1977... cit., II, p. 517.

42. Public transport and parking developments in Paris with particular reference to bus lanes - note of visit by G.L.C./L.T.B. offices in april 1969, avril 1969 (NA, MT/106/314).

43. Bus Lane for Brixton Road, dans GLC Press Office, 297, 3 juin 1969 (NA, MT/106/314).

44. Communication au Conseil de Paris du préfet de Paris et du préfet de police du 16 juin 1973 (APP, DA 830). Les «Lignes pilotes» lancées en 1973, formaient un réseau de sept lignes tobus à compter de 1970. Pour autant, la capitale britannique connaît des difficultés similaires à celles de Paris, notamment le manque de respect des couloirs.

\section{“IL NE RESTE PLUS QU'À QUADRILLER PARIS DE BANDES RÉSERVÉES ${ }^{46}$ »}

Ayant démontré leur capacité à améliorer le sort des autobus parisiens, les couloirs réservés devinrent dès lors la solution évidente et unique mise en avant par les pouvoirs publics. En 1973, les préfets de Paris, Jean Verdier, et de police, Jacques Lenoir, affirment qu'ils attachent «un intérêt primordial [...] à la sauvegarde et à la réhabilitation du réseau de transports en commun de surface ${ }^{47}$ ». L'administration tire ici les conséquences du raisonnement suivant :

Il apparaît clairement en 1973 que, dans des secteurs de plus en plus étendus et pour des périodes de la journée de plus en plus longues, la saturation de la voirie est telle que des accroissements très minimes de la circulation, ou des éléments imprévisibles (accidents, perturbations atmosphériques) suffisent à provoquer des paralysies quasi-totales. De ce fait, le métro garde une importance primordiale. Mais les investissements qui lui sont consacrés sont lourds et techniquement limités. La réhabilitation du réseau de surface de la RATP apparaît donc comme une nécessité ${ }^{48}$.

Visibles, mesurables et donnant des résultats quantifiables, assez peu coûteux à l'investissement et très peu coûteux à l'exploitation, les couloirs s'imposèrent dans le monde du transport parisien

principales intra muros, largement équipées de couloirs réservés, dotées de matériel neuf et identifiées par un logo spécifique. Il s'agissait de montrer la volonté d'innovation du réseau routier. L'opération rencontra un certain succès.

45. Les premiers axes équipés sont Park Lane, Vauxhall Bridge, Tottenham High Road et Brixton Road. Un essai de couloir à contresens sur $200 \mathrm{~m}$ avait été mené sur Berkeley Street en 1963, ce que la commission Lapébie avait pu constater sur place (note RATP/R/18249 du 30 décembre 1963 [ARATP, 1R 284]).

46. La théorie du domaine réservé... cit.

47. Communication au Conseil de Paris du préfet de Paris et du préfet de police du 16 juin 1973 (APP, DA 830).

48. Ibidem. 
jusqu'à en modeler la structure de pensée. Après diverses expériences malheureuses, comme celle des bus bleus, les pouvoirs publics pensent avoir enfin trouvé un outil efficace ${ }^{49}$. Le couloir fait donc office de principale politique en faveur des autobus à la fin des années 1960 et au tout début des années 1970, tout en rendant une ambition à un réseau qui s'était enlisé.

L'un des fondements de cette politique est publié par l'APUR en $1971^{50}$. Dans une étude marquante, il propose une restructuration profonde du réseau d'autobus intra muros en remaniant les itinéraires des lignes dans la zone définie par l'enceinte des Fermiers généraux (fig. 4). En se fondant sur une logique de calcul de temps des trajets porte à porte en autobus et en automobile, il démontre qu'une "utilisation rationnelle de la voirie» permet à un réseau d'autobus bénéficiant de $127 \mathrm{~km}$ de couloirs de doubler la capacité du réseau automobile existant ${ }^{51}$. Au-delà des arguments classiques fondés sur la surface occupée par chaque usager de la voirie, cette étude parvient à prouver l'efficacité d'un système de couloirs nouveaux $^{52}$. Elle affirme :

Le principe de la traversée des grands axes d'animation de la capitale par un réseau de transports collectifs de surface est la seule solution financièrement acceptable pour un développement de la ville, avec ses bureaux et ses commerces, son animation, dans de bonnes conditions de desserte ${ }^{53}$.

La logique du système proposé repose sur le rabattement des lignes d'autobus sur les axes équipés en couloir, ce qui présente plusieurs avantages (fig. 5). Il s'agit d'abord de supprimer les itinéraires dissociés du fait des sens uniques par la

49. Ainsi, la création d'un couloir réservé à contresens avenue Montaigne, long de $660 \mathrm{~m}$ et emprunté par deux lignes, soit 317 autobus par jour, a permis un gain de temps de $44 \%$ entre $7 \mathrm{~h}$ et $20 \mathrm{~h}$ et, plus précisément de $56 \%$ en heure de pointe. L'amélioration de la régularité est encore plus spectaculaire : le temps maximal de parcours passe de 17 à 6 minutes, le temps minimum de 3 minutes et 12 secondes à 2 minutes. Les kilomètres perdus pour difficulté de circulation ont d'ailleurs diminué de $18 \%$ sur les deux lignes, du simple fait de ce couloir. La RATP estime que, chaque année, sur l'ensemble des deux lignes, le trafic induit par ce couloir est de 185000 voyageurs, le temps gagné par les voyageurs de 76000 heures et le gain financier pour elle-même de 360 000 F (168 224 euros de 2006) (L. Servant, L'amélioration des transports urbains, expériences françaises et étrangères... cit., p. 90). Par ailleurs, en 1971, l'APUR estime que sur les cou- création systématique de couloirs à contresens. Ensuite, «le réseau étant simple présenterait le grand avantage psychologique d'être connu et de servir de point de repère, comme c'est le cas [...] pour le réseau du métropolitain ${ }^{54} »$. Enfin, les voies dégagées par les autobus sont laissées aux automobiles, ce qui accroît leur efficacité, tandis que la multiplication des autobus dans les couloirs permet d'éviter les critiques sur leur manque visible d'utilité. Il s'agit donc d'une solution dont bénéficient automobile et autobus, dans la mesure où un réseau aussi calqué sur la logique du métro peut encore être qualifié de réseau d'autobus. L'étude ne prend effectivement en compte que des indicateurs chiffrés (vitesse, régularité, capacité...) qui sont améliorés par ses propositions, mais ne laisse aucun espace à des considérations sur les pratiques des voyageurs, des machinistes ou des automobilistes, alors que le manque de respect des couloirs s'est avéré problématique. En ce sens, il s'agit encore essentiellement d'une étude de mécanique des fluides, très théorique. Elle touche pourtant aux questions d'espace public et d'usages dans la journée lorsqu'elle aborde, très succinctement, le problème des livraisons.

Cette étude de l'APUR fut à l'origine de la relance des couloirs au début des années 1970, puis du lancement des "Lignes pilotes», mais elle contribua à ne plus voir dans le réseau d'autobus qu'un réseau qui devait ressembler au métro, avec des infrastructures fixes et des vitesses élevées, ce que reprit à son compte la direction de la RATP, dominée par les ingénieurs du réseau ferré55. Ceci au risque de vider les quartiers et les axes secondaires, déjà lésés par la réorganisation de la Libération, de tout transport public et, afin de sauver l'autobus, de le transformer en un autre

loirs de plus de $400 \mathrm{~m}$, la vitesse moyenne est passée de 8 à $12,3 \mathrm{~km} / \mathrm{h}$ à la pointe (L. Coutinho, Étude de l'implantation dans le centre de Paris d'un réseau complet de couloirs réservés aux autobus, Paris, 1971, p. 16).

50. Ibid. Voir à ce sujet M. Flonneau, L'automobile à la conquête de Paris, 1910-1977... cit., II, p. 518.

51. L. Coutinho, Étude de l'implantation dans le centre de Paris d'un réseau complet de couloirs réservés aux autobus...cit., p. 3.

52. L'étude rappelle toutefois que les autobus, $1 \%$ des véhicules en circulation occupant $3 \%$ de la voirie, effectuent $24 \%$ des trajets (ibid, p. 7).

53. Ibid., p. 24

54. Ibid., p. 4.

55. Voir notamment Régie autonome des transports parisiens, 19491974, Paris, 1974, p. 4-5. 


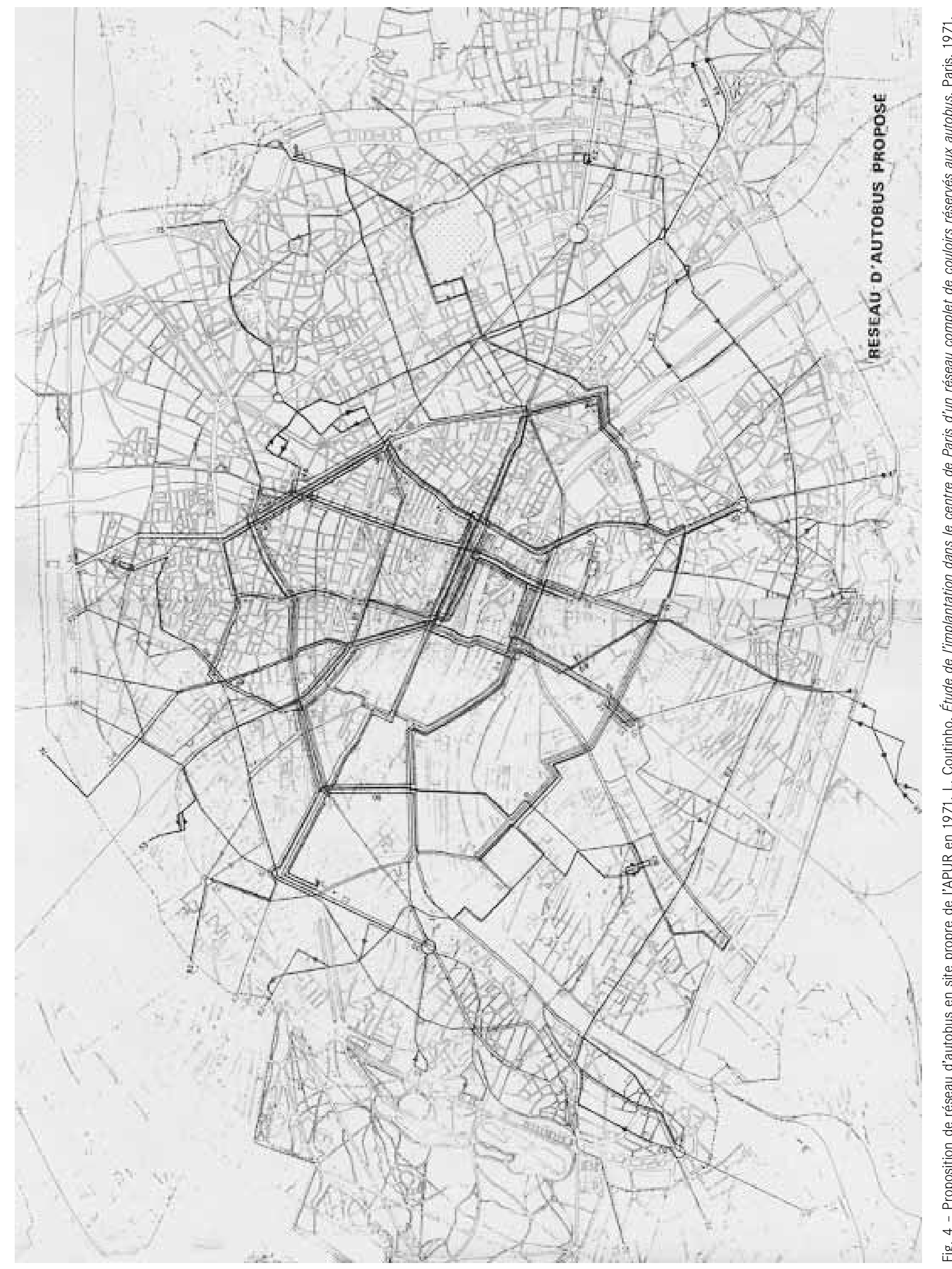




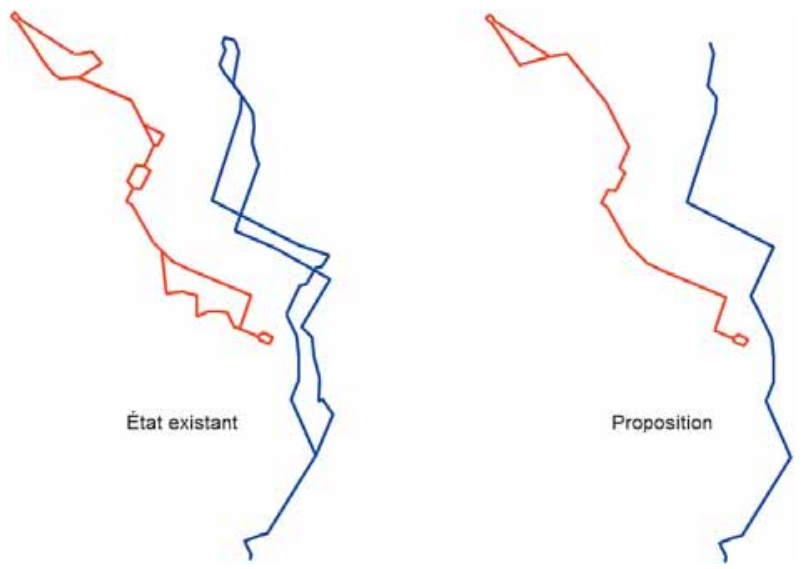

Fig. 5 - Exemples de modification d'itinéraires proposées par l'APUR en 1971 (lignes 84 [Place du Panthéon - Porte de Champerret] en rouge, et 67 [Place Pigalle - Porte de Gentilly] en bleu). L. Coutinho, Étude de l'implantation dans le centre de Paris d'un réseau complet de couloirs réservés aux autobus, Paris, 1971.

mode. Ainsi, le plan présenté en 1972 par Maurice Doublet, préfet de la région parisienne, s'appuie sur deux idées directement tirées du modèle du métro, selon la logique de l'étude de l'APUR: isoler presque totalement du reste de la circulation et des encombrements tous les parcours d'autobus à l'intérieur du périmètre réunissant les grandes gares d'une part; remanier le réseau pour éviter le plus possible les rues trop étroites d'autre part. Ce plan est par ailleurs véritablement ambitieux, puisqu'il prévoit des protections physiques des couloirs, par plots ou bornes basses et envisage des couloirs protégés situés au centre de la chaussée et non plus en rive ${ }^{56}$. France soir titre alors: «M. Doublet veut faire du réseau d'autobus un métro en plein air ${ }^{57}$ ".

Pour autant cette tentation de ne faire reposer la politique des transports intra muros que sur les seuls couloirs protégés est dénoncée de l'intérieur

56. Sur ce plan, dit plan bleu, voir notamment M. Doublet, Paris en procès, Paris, 1976, p. 107-108.

57. M. Doublet veut faire du réseau d'autobus un métro en plein air, dans France soir, 3 août 1972.

58. Note Budget de la RATP, accroissement de la vitesse des autobus de Maxime Mignon au préfet de police, ca. 1971 (APP, DA 830).

59. Communication sur la priorité à réserver aux transports en commun de surface, ca. 1971 (APP, DA 830).

60. Lettre du préfet de police au préfet de région du 5 février 1971 (APP, DA 830).

61. Ainsi Maurice Bourgoin, ancien directeur du réseau routier au début des années 1980, peut-il affirmer en 1992, après même de l'institution dès la fin des années 1960. Ainsi, le sous-groupe de la Préfecture de police en charge de ces questions préconise une politique très volontariste, visant au report modal de l'automobile vers l'autobus, en traçant un portrait cru de la situation : "la mise en service de nouveaux couloirs - et cela est prévu - n'apporte en tant que telle aucune amélioration à la situation actuelle» du fait de l'aggravation régulière des conditions de circulation et, surtout, d'un manque d'offre nouvelle sur le réseau d'autobus ${ }^{58}$. Au sein de la RATP, le couloir fait longtemps figure de panacée, ce que critique le préfet de police ${ }^{59}$. Pour lui, si «ces efforts sont nécessaires [...] il est bien évident qu'ils ne constituent qu'un très mince palliatif par rapport au problème de l'équilibre financier de la RATP», que seule une réforme tarifaire permettrait d'assurer ${ }^{60}$. Il faut attendre plusieurs années pour que la direction de la RATP, qui a vécu l'ouverture d'un premier couloir comme une véritable victoire politique, porte un regard nuancé sur cet objet $^{61}$.

Face aux réalisations en faveur de l'automobile à la même époque, le périphérique n'étant achevé qu'en 1973, aux projets à l'étude, notamment ceux de réseaux souterrains, et aux investissements massifs permettant la réalisation du RER, cette politique des couloirs réservés n'apparaît pas comme véritablement ambitieuse ${ }^{62}$. Le couloir est également un cache-misère dans la mesure où il repousse les problèmes le plus souvent en amont du linéaire protégé, c'est-à-dire au carrefour. Les difficultés s'accroissent avant de pénétrer dans le couloir, les véhicules particuliers étant confrontés à la nécessité de l'éviter, autobus et taxis étant contraints d'y pénétrer. Le couloir se rapproche ici de l'automobile, pour qui, comme l'écrit Pierre Sansot, «le carrefour démystifie la voiture. Elle perd ses pouvoirs s'être retiré des affaires, qu' «au total, cette solution présente tous les inconvénients d'une solution "tout ou rien." Limitons donc les couloirs à une partie du réseau, celle qui supporte de grandes densités de circulation, à titre d'exemple quand on a plus de soixante ou de cent bus à l'heure» (M. Bourgoin, «Propos liminaire», Séminaire Villes et transports, $4^{e}$ séance, 18 mars 1992).

62. La lecture de documents internes à la RATP retraçant l'histoire de ces années 1960-1970 permet de constater que les innovations mises en avant sont celles du réseau ferré, bien plus que celles du réseau routier (voir par exemple 50 ans d'histoire à la RATP, dans Entre les lignes, 1999, p. 12). 
imaginaires ${ }^{63} »$. Ainsi, malgré toutes ses qualités, la politique des couloirs se heurte à des difficultés croissantes, au fur et à mesure de son extension. Le couloir impose en effet de tenir compte des avis, souvent réticents, des riverains et livreurs, et de faire face au mécontentement des automobilistes, alors que des tronçons de moins en moins fréquentés sont équipés de couloirs se trouvant dès lors de plus en plus vides.

Solution ayant le mérite d'exister, d'être peu onéreuse et rapidement mise en ouvre, le couloir a sa place dans l'arsenal déployé par le gouvernement pour faire face à la crise énergétique de 1973. En effet, parmi les mesures annoncées par le Premier ministre le 30 novembre 1973, les premières concernent directement l'automobile : limitation des vitesses, interdiction des courses automobiles et incitation à l'utilisation des transports en commun. Les instructions que le ministre de l'Intérieur Raymond Marcellin envoie aux préfets sont claires : "renforcer la surveillance des couloirs réservés" est l'unique action recommandée, le couloir faisant toujours office de réflexe lorsqu'il s'agit des transports en commun ${ }^{64}$.

Toutefois, l'effet réel des couloirs n'est que l'enrayement de l'enlisement, à Paris comme en banlieue, ce qui incite à relativiser leur efficacité et surtout à prendre en considération les difficultés rencontrées par la circulation en amont de chaque couloir (fig. 6). Dès lors, leur impact ne serait-il pas ailleurs que sur la voirie?

\section{LE COULOIR, UNE AVANCÉE CONCEPTUELLE PLUS QUE MATÉRIELLE?}

Aux progrès réalisés sur la voirie elle-même en termes de vitesse et de régularité, teintés de difficultés nouvelles, les accidents, vient effectivement s'ajouter un volet extrêmement important sur lequel les couloirs marquent une véritable

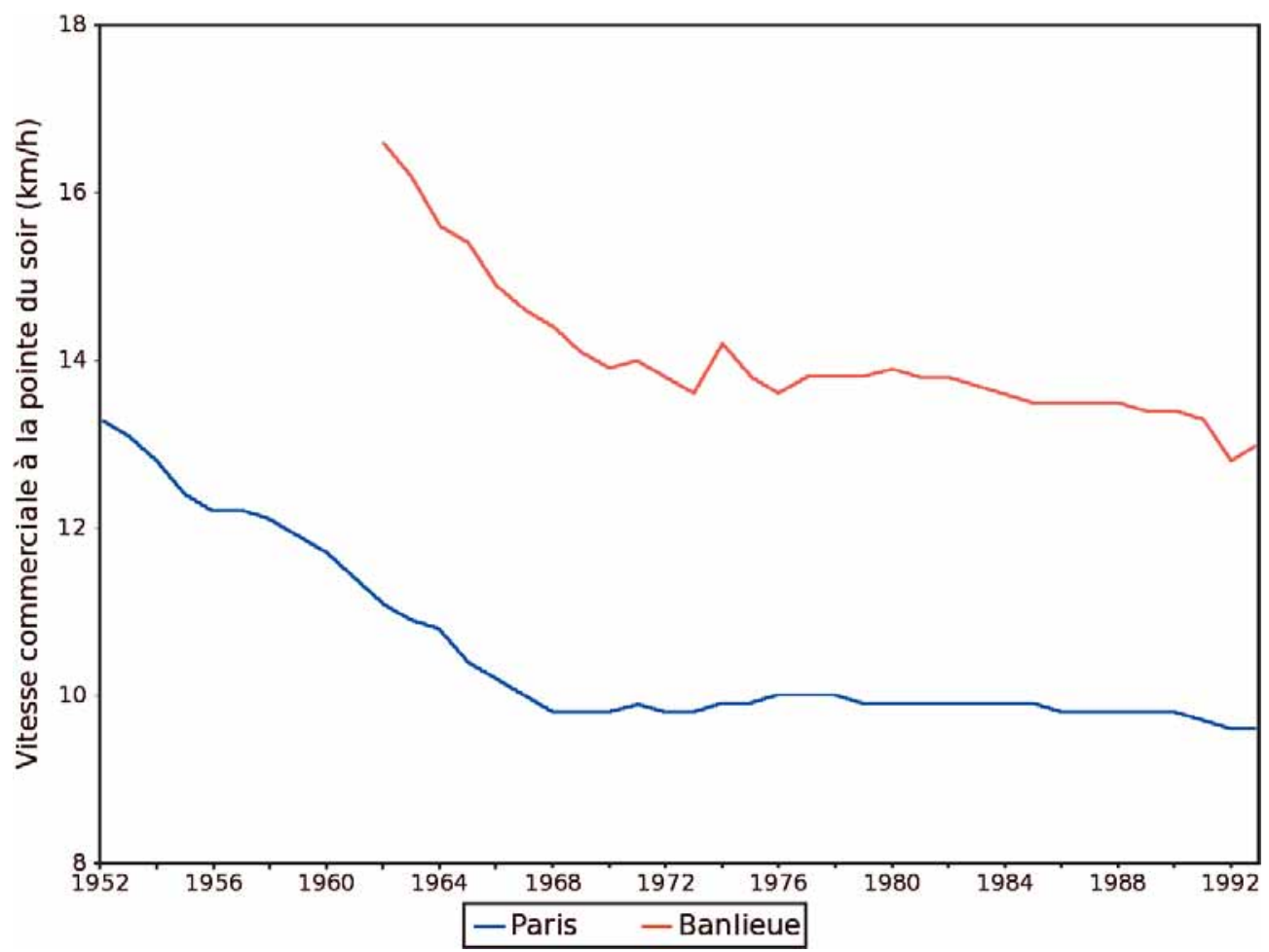

Fig. 6 - Évolution de la vitesse commerciale des autobus de Paris et de banlieue à la pointe du soir (1952-1993). Statistiques annuelles de la RATP, 1993.

63. P. Sansot, Poétique de la ville, Paris, 1996, réédition, 2004, p. 280.
64. Télégramme du ministre de l'Intérieur aux préfets $73570 \mathrm{du}$ $1^{\text {er }}$ décembre 1973 (ARATP, IR 185). 
avancée : l'image de l'autobus. Ce mode vieillissant, que les médias ne mettaient en scène que pour dénoncer les grèves ou les embouteillages qu'il provoquait, faisait de nouveau la une des journaux en présentant enfin une nouveauté durable $^{65}$. Lui, qui a souffert de n'avoir fait que d'obscurs progrès techniques au cours des années 1950, pouvait mettre en avant un objet visible du grand public comme des cercles techniques, ce qui était un des objectifs de la direction de la RATP, notamment le directeur du réseau routier Louis Meizonnet, objectif que ne partageait pas la Préfecture de police, pour qui il suffisait de faire respecter les couloirs, sans chercher de retentissement particulier. Or le poids de l'opinion publique est primordial. La rupture de 1968 et les évolutions du début des années 1970 ont ainsi permis une généralisation du couloir, dont la création s'est toutefois faite dans un contexte a priori peu favorable, les opposants, tenants de l'automobile, instrumentalisant les premiers accidents qui y survinrent ${ }^{66}$.

Comme toute innovation en matière d'aménagement, les bandes réservées suscitent effectivement les plus vives contestations lorsqu'arrivent les premiers accidents graves. C'est donc après la mort d'une piétonne dans le tout nouveau couloir à contresens de la rue de Rivoli, le 11 octobre 1967, qu'une campagne à l'encontre de ces objets est menée par une partie de la presse qui trouve enfin un argument imparable pour contester l'idée même de couloir ${ }^{67}$. Les critiques sont cinglantes, les journaux titrant sur «les couloirs de la mort ${ }^{68}$ ».

65. Comme l'écrit Combat à l'ouverture du premier couloir, «les autobus, près de la retraite, retrouvent une nouvelle jeunesse en pouvant avancer à grande vitesse" ( $L a$ théorie $d u$ domaine réservé... cit.). Voir également, pour ce qui est des images filmées, FI, Actualités Gaumont, novembre et décembre 1975.

66. Pour Alfred Sauvy comme pour Louis Servant, l'opinion publique est un des facteurs-clés à prendre en compte dans le projet (A. Sauvy, Les quatre roues de la fortune, Essai sur l'automobile...cit., p. 175; L. Servant, L'amélioration des transports urbains... cit., passim).

67. Une femme de 35 ans a voulu traverser la rue de Rivoli à $3,50 \mathrm{~m}$ à gauche du passage protégé. Ne connaissant pas le nouvel équipement à contresens, elle a été surprise par l'arrivée, sur sa gauche, d'un autobus de la ligne 85, qui n'a pu l'éviter et l'a finalement renversée. Le couloir avait été installé le 2 octobre 1967 (voir Le feu rouge ne la protégeait pas de l'autobus venant à contre-courant, dans France soir, 12 octobre 1967).
Ce fait divers tragique semble donner raison à Maurice Papon qui n'avait pas retenu l'idée des couloirs à contresens initialement proposée par la RATP. En réalité, il pose surtout la question de l'apprentissage que doivent faire les usagers des évolutions de l'espace public et, plus généralement, de l'accompagnement de l'innovation. Pressés par un deuxième accident, moins grave, en décembre 1967, et fidèles à la logique d'équipement de l'espace public, plus qu'à celle de l'apprentissage ou de la police, les pouvoirs publics installent rapidement des barrières temporaires, puis des chaînes, le long de ce couloir à contresens, renforçant toujours plus une séparation visible des flux ${ }^{69}$. Le jeu sur la scène médiatique a pu donc s'avérer dangereux pour les couloirs et, au-delà de la sécurité, l'objet vient troubler un état de fait en s'inscrivant dans une logique nouvelle de maîtrise de la place de l'automobile en ville. Tandis que les piétons sont victimes involontaires de la lutte engagée entre automobiles et autobus.

L'ouverture des couloirs d'autobus s'inscrit dans un ensemble nouveau de "dispositifs de reconquête sur l'automobile», déployés pour lutter contre la «tripe automobile» que le Préfet de police juge relever des valeurs nationales ${ }^{70}$. Le plus symbolique est la création de zones piétonnes d'où l'automobile est exclue, notamment place du Tertre en 1964. Le plus contraignant est probablement le stationnement payant, mis en place à compter d'octobre 1971 sur un secteur central limité $^{71}$. Pour sa part, la création de couloirs supprime des places de stationnement automobile et,

68. Expression récurrente de la presse parisienne, notamment présente dans Nous avons gagné!, dans Paris jour, 12 octobre 1967. Voir également Les couloirs de circulation à contresens en accusation. Une femme est morte victime des "améliorations" de la circulation parisienne, dans L'aurore, 11 octobre 1967, article présentant les lieux du crime, avec un dessin du corps de la victime à terre, un autobus arrivant en arrière-plan. Voir aussi Tuée par le 85 dans le "couloir" de la rue de Rivoli, dans Paris jour, 11 octobre 1967.

69. Voir La distraction d'une jeune institutrice à l'origine d'un second accident dans les "couloirs à contresens" pour autobus de la rue de Rivoli, dans L'aurore, 4 décembre 1967.

70. M. Flonneau, L'automobile à la conquête de Paris, 1910-1977... cit., II, p. 524; Communication sur la priorité à réserver aux transports en commun de surface, ca. 1971 (APP, DA 830).

71. Voir M. Couvrat-Desvergnes et T. du Crest, Il y a 30 ans, les premières mises en place du stationnement payant sur voirie, dans TEC, 164, 2001, p. 10-17. 
plus rarement, des files de circulation, tout en rendant plus difficile l'accès aux garages ou stationsservices en rive. Le couloir à contresens est un élément typique de la reconquête contre l'automobile et les sens uniques qu'elle a imposés. Il permet d'associer à nouveau les itinéraires aller et retour d'une même ligne. Mais il peut également s'agir de retrouver un itinéraire dégagé, comme la rue de Rivoli, alors que l'itinéraire dissocié se trouvait ralenti par la congestion des voies étroites empruntées auparavant.

Cette politique attise donc la colère de porteparole de l'automobile individuelle. Comme l'explique Combat, "certains automobilistes 'privés' qui utilisent leur voiture dans Paris considèrent la création des 'pistes' comme une entrave à la liberté de circuler et même à la liberté tout court. Les plus violents disent qu'ils refuseront d'être voués au métro et à l'autobus ${ }^{72} »$. Plusieurs réclament la construction de parcs de stationnement aux portes de Paris, mais "si certains automobilistes se résignent à devoir emprunter un jour les transports en commun dans le centre de la capitale, d'autres qui font profession d'individualisme, refusent $d^{\prime}$ envisager cette hypothèse ${ }^{73}$ ». La presse automobile appelle à la mobilisation contre ces couloirs qui sont «un ballon sonde [...]. L'on guette les réactions des automobilistes. Si ceux-ci se montrent passifs, nul doute qu'une nouvelle catégorie de privilèges verra le jour [...]. La fin de la féodalité avait vu celle des privilèges, le $\mathrm{XX}^{\mathrm{e}}$ siècle les verra renaître ${ }^{74} »$.

Au-delà de ces contestations informelles, le Syndicat national des automobilistes (SNA) engage un recours en annulation de l'arrêté préfectoral créant le couloir dès mars 1964 en se fondant sur l'illégalité supposée de l'attribution d'une partie de la chaussée à un type de véhicules spécifiques ${ }^{75}$. Le SNA avait obtenu satisfaction en contestant des arrêtés de stationnement réservé.
Le conseiller Breton avait soulevé cette question dès le 19 janvier 1964, ce à quoi le préfet de police avait répondu en fondant la légitimité légale des couloirs sur leur cohérence avec les lignes d'action définies en faveur des transports publics, la moindre chaussée par personne occupée par les voyageurs en autobus, qui ne pouvaient donc être qualifiés de privilégiés, et enfin sur deux éléments plus contestables : le caractère temporaire des couloirs, qui fut progressivement abandonné, et leur positionnement particulier vis-à-vis des commerces et riverains, qui ne put être tenu au fur et à mesure de leur généralisation ${ }^{76}$. Le recours du SNA fut finalement rejeté par le Conseil d'État, ce qui n'empêcha pas l'intensification des critiques lors de la relance du programme par Maurice Gri$\operatorname{maud}^{77}$.

Mais au-delà de ces contestations venues du monde de l'automobile, les élus parisiens maintiennent leur appui au réseau d'autobus et votent l'extension des couloirs. Cette position est notable puisque les intérêts commerçants, classiquement ressentis comme puissants par les élus locaux, se mobilisent également contre les couloirs, la Chambre de commerce de Paris s'impliquant dans la contestation de ces nouveaux aménagements. Toutefois, des réticences locales, dans les circonscriptions, sont sensibles, même si les élus sont d'accord sur le principe des couloirs ${ }^{78}$. En banlieue, où le jeu institutionnel est plus complexe, les couloirs sont d'ailleurs d'autant plus difficiles à créer. La volonté politique peut toutefois s'appuyer sur le discours du préfet Doublet qui n'hésite pas à déclarer $\mathrm{qu}^{\prime}$ «il faut avoir le courage d'être impopulaire pour n'être pas, demain, accusé d'imprévoyance. [Il faut] choisir, dans les zones denses, entre l'autobus et la voiture individuelle ${ }^{79}$ ".

Le courage des pouvoirs publics, notamment de Maurice Grimaud, s'avère payant. Contre toute
72. L'expérience des "pistes» pour autobus est concluante déclare la RATP après quelques jours d'essai sur les quais... cit.

73. Ibidem

74. La lutte contre les privilèges des couloirs de circulation. Le SNA attaque le Préfet de Police en excès de pouvoir... cit.

75. Il dénonce "un arrêté illégal. La chaussée appartient à tout le monde" (Ibidem).

76. Liaisons, 3 février 1964

77. Comme l'a montré Mathieu Flonneau, L'équipe attaqua Maurice Grimaud, notamment dans son édition du 26 sep- tembre 1968, l'accusant alors de créer 17 couloirs d'autobus aux dépens de 1000 places de stationnement; ce à quoi le préfet de police répondit en expliquant qu'il fallait raisonner sur des modes de transport modernes et non plus sur "l'autobus à papa" (M. Flonneau, L'automobile à la conquête de Paris, 1910-1977... cit., II, p. 516)

78. Le Conseil de Paris a notamment imposé le démontage des bordurettes de protection qui avaient été testées avenue Franklin Roosevelt en 1973.

79. Le Figaro, 15 novembre 1972. 
attente, et après quelques années d'appropriation, il semble que l'opinion publique présente une adhésion massive aux couloirs réalisés ${ }^{80}$. Comme l'écrivent les préfets de Paris et de police, «il apparaît une large convergence d'opinions favorables sur l'utilité des couloirs de circulation. Un autre enseignement qui mérite de retenir l'attention est l'absence d'opposition entre les automobilistes et les usagers des autobus ${ }^{81}$ ». Le plus inattendu est que les commerçants, réputés hostiles aux couloirs, se montrent, après leur installation, plutôt favorables à ces objets, à l'image de ce qui s'est passé avec le stationnement payant ${ }^{82}$.

Reste à savoir si le couloir est un objet suffisant pour briser le cercle vicieux de l'enlisement de l'autobus. Comme l'écrivait Alfred Sauvy, "pour briser ce cercle, il faut [...] des gestes d'une extrême énergie..$^{83}$ " Certes, le statut du couloir impose aux automobilistes de respecter les autobus, sous peine d'amende ${ }^{84}$. Encore faut-il que la verbalisation ait lieu et, pour l'efficacité du dispositif, que le véhicule soit enlevé. Ce qui nécessite une volonté politique de long terme des forces de police, qui ne fut qu'épisodiquement au rendez-vous ${ }^{85}$. La RATP parvint toutefois à obtenir l'autorisation pour certains de ses agents assermentés de procéder à des constats d'infraction le long des couloirs réservés et aux abords des points

80. Une enquête de l'IFOP sur un échantillon des habitants de l'ancien département de la Seine et des commerçants du boulevard Saint-Germain en 1972-1973 fait ressortir les résultats suivants (communication au Conseil de Paris du préfet de Paris et du préfet de police du 16 juin 1973 [APP, DA 830]) :

- 75\% des usagers sont favorables à l'extension des couloirs réservés;

- 77\% des habitants de l'ancienne Seine leur attribuent une utilité véritable et leur destinent en premier lieu les autobus $(89 \%)$, les ambulances et les services de secours $(78 \%)$ et, enfin, les taxis $(69 \%)$;

- $65 \%$ des personnes interrogées pensent qu'il pourrait y avoir un danger pour les piétons;

- 34\% des personnes interrogées pensent qu'il pourrait y avoir une gêne pour les commerçants riverains;

- $75 \%$ des habitants sont favorables à l'extension dans un sens;

- $51 \%$ des habitants sont favorables à l'extension dans les deux sens;

$-81 \%$ des usagers sont favorables à l'extension;

- 59\% des usagers sont favorables à l'extension dans un sens;

- $74 \%$ des usagers et $53 \%$ des usagers occasionnels pensent qu'une telle mesure les inciterait à recourir plus souvent à ce mode de transport. d'arrêt ${ }^{86}$. C'est donc un accroissement de sa légitimité que la RATP doit aux couloirs d'autobus, puisqu'elle obtient une part des pouvoirs de police qu'elle a souvent tenté d'usurper.

Plus généralement, le couloir réservé présente l'intérêt de poser concrètement et publiquement plusieurs questions théoriques. Quel doit être l'usage de l'espace public? Peut-il être réservé en partie à certains usagers? Comment moduler dans le temps les usages d'un espace contraint? En réalité, derrière l'autobus se cachent d'autres modes, taxis, livreurs, puis vélos, qui profitent de cette situation pour également apparaître au grand jour et se distinguer d'un flux automobile qui semblait jusque-là homogène dans ses besoins exprimés. Comme l'a expliqué Maurice Bourgoin, «les problèmes de congestion débouchent in fine sur une idée centrale selon laquelle la voirie est un marché ${ }^{87}$ ». Le couloir fait également ressortir les tensions à l'intérieur de l'institution, entre les services en charge des transports et ceux en charge de la circulation. Il met en exergue la nécessité d'une approche globale de la politique des transports et d'une unité unique responsable de cette politique, comme cela se constitue alors en province.

Enfin, dans les débats entre concepteurs, exploitants et pouvoirs publics, l'autobus fait un pas gigantesque lors de la création de couloirs : il

81. Idem.

82. Comme l'écrivent les préfets, «la quasi-totalité des commerçants intéressés jugent très utiles ou assez utiles les couloirs réservés. Cette proportion tombe à 33\% chez les non riverains, qui paraissent redouter des difficultés de livraison et une diminution de leur clientèle" (idem).

83. Sauvy, Les quatre roues de la fortune, Essai sur l'automobile... cit., p. 172.

84. Un flou juridique demeurait toutefois sur les possibles sanctions encourues par les véhicules non-autorisés empruntant ces couloirs. En 1969, l'amende pour l'utilisation d'un couloir était de $30 \mathrm{~F}$ (30,00 euros de 2006) mais s'exposait à contestation.

85. Citons ici une circulaire de 1973 annonçant un renforcement de l'intérêt porté aux transports en commun : «ces dispositions traduisent la volonté des pouvoirs publics de privilégier la circulation des transports collectifs et de remédier aux difficultés qu'ont [...] les forces de police urbaine à surveiller, autant que nécessaire, ces axes et emplacements réservés» (circulaire des ministres de la justice, de l'Intérieur et des transports aux préfets du 14 mars 1973 [ARATP, 1R 148]).

86. Les agents chargés de la surveillance des itinéraires (ACSI) sont autorisés par l'arrêté ministériel du 12 mars 1973 s'appliquant à Paris et dans quelques villes de province.

87. M. Bourgoin, Propos liminaire... cit. 
présente enfin une infrastructure. Ce mode qui a longtemps été perçu comme souple et sans besoin d'aménagements spécifiques rend explicite le biais de cette conception, ou du moins son obsolescence. Face au réseau ferré, le réseau routier peut désormais présenter des axes visibles, nécessitant une conception de la voirie laissant une place à l'autobus. Mais le couloir, délimité par une bande de peinture, n'est pas une infrastructure véritablement nouvelle et ne peut donc pas jouer sur l'imaginaire de l'aménagement du territoire, encore fort avant le milieu des années 1970. Il présente également aux voyageurs une matérialisation de ses itinéraires précieuse et inhabituelle. La spécificité du réseau routier est toutefois d'être toujours bien plus confronté aux questions d'insertion urbaine et notamment de concertation en vue de l'établissement d'un couloir nouveau, avec riverains, commerçants ou livreurs, ce que le creusement du tronçon central du RER ne nécessite pas.

$C^{\prime}$ est ainsi que le couloir finit par s'identifier à l'autobus lui-même. C'est du moins la volonté de la direction de la RATP qui lance, au milieu des années 1980, une publicité intitulée Couloirs de bus présentant deux zèbres aux couleurs des tickets d'autobus courant sur la droite d'un troupeau de gazelles et dont le slogan est : "à chacun son territoire ${ }^{88}$ ". Paris voit même naître, le 9 mars 1970, une voie entièrement réservée aux autobus, la petite et méconnue rue de la tacherie ${ }^{89}$. Cas exceptionnel, qui incarne à merveille l'ambition limitée des concepteurs des couloirs.

Mais ces avancées immatérielles en faveur de l'autobus ne sont pas sans dangers pour lui. Réserver un espace à des usagers spécifiques conduit chaque mode à se trouver légitime à réclamer sa propre voie, pour des raisons d'utilité publique ou d'efficacité collective. Autre problème naissant, plus interne à la RATP, l'instauration d'une hiérarchie entre les lignes équipées de couloirs et les autres. Cette logique, renforcée par l'opération des "Lignes pilotes», est une des prin-

88. Voir PRATP, 165 872. Cette thématique est d'ailleurs fréquente dans la communication de la RATP. Des campagnes publicitaires récurrentes au cours des années 1970 incitèrent à un meilleur respect des couloirs, ce qui traduisait la faiblesse de l'objet face aux pratiques réelles (voir PRATP, 130 932, 130923 et 132782 à 132 787).

89. Cette innovation est permanente, du quai de Gesvres à l'avenue Victoria, sur $50 \mathrm{~m}$. Le projet de réservation de la cipales problématiques auxquelles se confronte l'autobus du dernier quart du $\mathrm{XX}^{\mathrm{e}}$ siècle.

Le couloir est un objet porteur d'enjeux sociaux et moraux que cache difficilement une représentation purement technique, attachée au choix des axes à équiper. Il est d'abord un objet révélateur, rendant visible ce qui ne l'était que très partiellement jusque-là : le réseau d'autobus. Audelà des itinéraires, il expose aux yeux de tous, voyageurs, piétons ou automobilistes, l'exploitation du réseau, ses irrégularités et ses fréquences. Ses promoteurs ont pris le risque de l'image des couloirs vides, avant de la combler en y acceptant les taxis, aux dépens de la vitesse des autobus euxmêmes. La pression sociale pour renforcer la légitimité de ces objets a donc été plus forte que les besoins techniques de l'exploitation du réseau. En ce sens, le couloir n'est pas qu'un simple révélateur, il est également un actant, pour reprendre le vocabulaire de Bruno Latour. Par un effet cliquet, il s'impose à tous, rendant impossible tout retour en arrière par sa présence physique et son influence sur la conception même des modes.

Ces bandes de roulement rendent explicites les tensions de l'espace urbain, en matérialisant la rivalité entre les modes. Ils concrétisent, d'abord par une bande de peinture, puis, au début du $\mathrm{XXI}^{\text {e }}$ siècle par des séparateurs physiques, une situation que des années de développement automobile ont rendue implicitement délicate. Ils signifient clairement l'opposition entre intérêts automobiles et intérêts des transports collectifs, et, de ce fait, scellent le divorce entre ces modes, engagé autour de la Deuxième Guerre mondiale. Les deux modes s'étant séparés, le couloir incarne l'arbitrage pris entre eux par les pouvoirs publics pour le partage d'un espace restreint ${ }^{90}$. Le couloir présente ainsi la vertu de concrétiser une politique : l'éternelle priorité aux transports en commun, promise par de nombreux décideurs, se trouve là visible sur la chaussée, que ce soit ou non la solution la plus efficace. Une réforme tari-

rue du faubourg Montmartre aux autobus est en revanche abandonné à la fin des années 1970.

90. Comme l'écrit Louis Servant dès 1978, "si les notions de planification et d'urbanisme sont relativement anciennes, la notion de planification des transports urbains n'est apparue que récemment, avec la saturation de la voirie engendrée par le développement de la circulation automobile» (L. Servant, L'amélioration des transports urbains ... cit., p. 5). 
faire, qui n'intervint qu'à partir de 1975 avec la Carte orange, eût peut-être eu des résultats très probants, mais ne présentait pas l'avantage d'être aussi lisible. Partage de l'espace, il est également un partage du sens. Comme l'écrit Marc Desportes : «La question posée par l'aménagement urbain contemporain est celle du partage du sens avec autrui. Tout aménagement, même le plus pauvre, peut devenir le support d'une signification. [...] Mais le sens ainsi élaboré est personnel et ne s'articule plus sur des marques spatiales reconnues collectivement ${ }^{91}{ }$.

L'ambiguïté de l'objet est caractéristique d'une société qui n'accepte qu'en partie la nécessité d'une évolution de ses pratiques de mobilité. En fondant le report modal sur la répartition spatiale, il est effectivement un avatar très simple de la théorie de l'urbanisme moderne prônant une séparation des flux. Or simultanément, c'est l'application de ces mêmes principes de la charte d'Athènes qui a conduit à la ruine du réseau de surface $^{92}$. Dès lors, ne s'agit-il pas d'un objet dont les origines conceptuelles sont dépassées ou du moins contestées à l'époque où il se généralise, c'est-à-dire après 1968? En suivant la critique portée par Richard Sennett envers l'architecture et l'urbanisme fonctionnels, qu'il accuse d'associer "esthétique de la visibilité et isolement social», le couloir d'autobus peut être considéré comme un parfait exemple de l'appauvrissement des aménités d'un espace public réduit à n'être qu'un espace circulatoire où les uns et les autres se voient attribuer des domaines privés ${ }^{93}$. "L'espace public est devenu un dérivé du mouvement», écrit Richard Sennett, ce qui reflète l'influence du métro, qui crée son propre espace, dédié à son propre mouvement ${ }^{94}$. Comme l'explique Pierre Sansot, les Trente Glorieuses ont désacralisé l'espace urbain qui peut dès lors accueillir tout type d'objets, y compris les plus fonctionnels, tels les couloirs d'autobus ${ }^{95}$.

91. M. Desportes, Paysages en mouvement... cit., p. 368.

92. Voir Le Corbusier, La charte d'Athènes, Paris, 1943, réédition, 1957, passim.

93. R. Sennett, Les tyrannies de l'intimité, Paris, 1979, p. 22.

94. Ibid., p. 23.

95. P. Sansot, Poétique de la ville... cit., p. 68.

96. B. Latour, Petites leçons de sociologie des sciences, Paris, 1993, réédition, 2006, p. 25-32.

97. Le cas des vélos, qui ne furent autorisés à rouler dans cer-
Comme tout dispositif de discrimination positive, le couloir est autant un élément en faveur de l'autobus qu'un élément le stigmatisant et suscitant la jalousie. Il revêt ainsi un caractère moral très net. Il rappelle la ceinture de sécurité dont Bruno Latour a analysé les missions ${ }^{96}$. Il distingue les modes dont la légitimité à occuper l'espace public est stable ou croissante de ceux dont la légitimité chancelle. Les véhicules d'urgence, qui jouissent d'une indéniable utilité a priori, partagent les couloirs avec autobus et taxis dont la légitimité se fonde sur la notion de transport en commun ${ }^{97}$. C'est cette différence, attachée à la surface occupée par voyageur nettement plus qu'à des considérations environnementales, qui renvoie les automobiles individuelles de l'autre côté de la bande de peinture. Les pouvoirs publics montrent donc du doigt les individualistes, punis par l'encombrement de la voirie, pour les opposer aux collectivistes, récompensés par une circulation améliorée. Mais cette circulation n'est améliorée que par rapport à ce qu'elle était en l'absence de couloir, pas nécessairement meilleure que celle de la chaussée banalisée. C'est une des faiblesses du dispositif. Une lecture sociale du couloir conduirait à distinguer deux clans, ceux qui ne peuvent rouler en automobile, et qui donc prennent l'autobus, mêlés à ceux qui peuvent s'offrir le taxi, opposés à une masse d'automobilistes écrêtée des deux côtés de l'échelle sociale. Reste que le but avoué de cette mesure est un report modal qui doit remplir le couloir et vider la chaussée banalisée. L'objet ne peut donc être théoriquement que provisoire, que son efficacité se traduise par un fort report modal, ou que son inefficacité conduise à sa suppression. Enfin, le couloir mérite d'être rapproché de la théorie du surcode développée par Lucien Sfez. Il est possible de voir dans cet objet un signe annexe, conçu en fonction de ce que chacun des acteurs en présence pense de l'autre et de ce que l'autre peut comprendre ${ }^{98}$. Le couloir incarne cet échec du dialogue complexe et

tains couloirs qu'au début du XXI ${ }^{\text {e }}$ siècle est significatif : ils avaient alors acquis une légitimité nouvelle, liée aux préoccupations environnementales.

98. À l'image de l'application qu'en ont faite Jean-Marc Offner et Agnès Sander à propos de l'opération des années 19801990 de restructuration du réseau d'autobus de banlieue Autrement Bus (J.-M. Offner et A. Sander, Les points-clés d'Autrement bus : des théories à la pratique. Analyse de la mise en auvre d'une innovation à la RATP, Paris, 1990, p. 8-9). 
cette nécessaire simplification, fondée sur la représentation que chacun se fait des autres acteurs. Comme l'explique Lucien Sfez, ce type d'objet finit par l'emporter et par dominer la rationalité : la politique des transports de surface se résume donc au couloir, qui impulse néanmoins une nouvelle dynamique.

\section{D'UN COULOIR POUR L'AUTOBUS \\ À UN COULOIR CONTRE L'AUTOMOBILE?}

Ainsi les années 1960 parisiennes ne peuventelles se résumer aux nouvelles autoroutes et aux projets de RER destinés à développer une mobilité sans limites. En effet, la priorité en faveur des transports en commun trouve à cette même époque une concrétisation tangible, ce mouvement étant accentué, de façon visible, au cours des années 1970. Le déploiement de ces couloirs réservés nous conduit à deux remarques. La première est que ces couloirs, destinés à faciliter la circulation des autobus, ont été rapidement ouverts à d'autres véhicules, ce qui en a réduit l'efficacité initiale. Se trouve ainsi illustrée l'hypothèse plus générale selon laquelle tout nouvel objet destiné à accroître la mobilité se heurte à des dispositifs ou dispositions destinées à le freiner. Pensons ici à l'invention des arrêts fixes, qui a suivi la motorisation des omnibus à la Belle Époque, quitte à en réduire la fluidité et l'accessibilité. Le même phénomène n'est-il pas à l'œuvre dans la conception même d'un dispositif de discrimination positive? Chacun développe son propre argumentaire et obtient son propre objet, ce qui ne débouche probablement pas sur la fluidité la plus parfaite.
L'autre remarque conclusive tient à la place qu'occupe l'outil, ici le couloir, dans le raisonnement des techniciens et de la sphère politique et, plus largement, dans le débat public sur les transports de la région parisienne. L'outil procure une visibilité nouvelle à des questions jusque-là peu médiatisées, suscite la controverse mais contribue également à fortement modeler la vision développée par différents acteurs sur le sujet, notamment les responsables de la RATP. Jusqu'à la fin du siècle, le couloir, fort de son pouvoir médiatique, est la solution unique, tout en connaissant une contestation constante de la part d'automobilistes peu respectueux de sa logique. Ainsi, le maire est conduit à renforcer cet objet, par l'ajout d'un dispositif concret de séparation des flux. Si le même effet de résonance est notable pour les aménagements mis en place par Jean Tiberi en 1999, puis, à une échelle plus vaste, par Bertrand Delanoë à partir de 2001, il s'agit bien à nouveau de discuter de la place et du rôle de l'objet sur l'espace public. Effectivement, le déploiement de couloirs protégés par une bordurette marque une nouvelle étape des relations entre autobus et automobile qui peut se comprendre comme une phase d'instrumentalisation de l'autobus contre l'automobile. Le couloir, séparateur visible de deux catégories de véhicules sur l'espace de voirie, devient ainsi le médiateur entre deux camps sur l'espace politique, le pouvoir municipal choisissant une politique de l'objet plus qu'une politique de la règle, que pouvait constituer le choix du péage urbain ou tout simplement un meilleur respect du couloir séparé par une bande de peinture.

Arnaud Passalacqua 